\title{
Slow Oscillation in Non-Lemniscal Auditory Thalamus
}

\author{
Jufang He \\ Department of Rehabilitation Sciences, The Hong Kong Polytechnic University, Hung Hom, Kowloon, Hong Kong, China
}

In the present study, we investigated the oscillatory behavior of the auditory thalamic neurons through in vivo intracellular and extracellular recordings in anesthetized guinea pigs. Repeated acoustic stimulus and cortical electrical stimulation were applied to examine their modulatory effects on the thalamic oscillation. The time course of the spike frequency over each trial was obtained by summing all spikes in the onset period and/or the last time period of 100 or $200 \mathrm{msec}$ in the raster display. Spectral analysis was made on the time course of the spike frequency. A slow-frequency oscillation ranging from 0.03 to $0.25 \mathrm{~Hz}$ (mean $\pm \mathrm{SD}, 0.11 \pm 0.05 \mathrm{~Hz}$ ) was found in the medial geniculate body (MGB) together with a second rhythm of 5-10 Hz. The oscillation neurons had a mean auditory response latency of $17.3 \pm 0.3 \mathrm{msec}$, which was significantly longer than that of the non-oscillation neurons in lemniscal MGB $(9.0 \pm 1.5 \mathrm{msec}, p<0.001$, ANOVA) and similar to the non-oscillation neurons in the non-lemniscal MGB (17.6 $\pm 5.4 \mathrm{msec}, p=0.811)$. They were located in the non-lemniscal nuclei of the auditory thalamus. Cortical stimulation altered the thalamic oscillation, leading to termination of the oscillation or to acceleration of the rhythm of the oscillation (the average rhythm changed from $0.07 \pm 0.03$ to $0.11 \pm 0.04 \mathrm{~Hz}, n=8, p=$ $0.066, t$ test). Acoustic stimulation triggered a more regular rhythm in the oscillation neurons. The present results suggest that only the non-lemniscal auditory thalamus is involved in the slow thalamocortical oscillation. The auditory cortex may control the oscillation of the auditory thalamic neurons.

Key words: corticofugal modulation; medial division of the medial geniculate body; electrical stimulation; in vivo intracellular recording; dorsal division of the MGB; stimulus-induced oscillation

\section{Introduction}

Several commonly observed rhythms are related to the thalamocortical loops: $\beta$ wave, spindles, delta oscillation, and the slow oscillation of $0.2-0.5 \mathrm{~Hz}$ (Steriade et al., 1993a-d; Steriade, 2001a; Steriade and Timofeev, 2003). The spindle and slow oscillations have been shown in neurons in the dorsal lateral geniculate body and in the ventroposterior nucleus of the thalamus, respectively, with slice preparation (von Krosigk et al., 1993; Golshani and Jones, 1999). Both the cortex and the thalamus have an intrinsic capacity to generate oscillations of different frequencies (Steriade et al., 1993e; Destexhe et al., 1993; Golshani and Jones, 1999; Bal et al., 2000), but the slow-wave oscillations in the EEG are more likely to involve the corticothalamocortical loop (Steriade et al., 1993a-d). Cortical feedback controls the thalamic oscillation via the thalamic reticular nucleus (TRN) (Huntsman et al., 1999; Bal et al., 2000; Blumenfeld and McCormick, 2000; Golshani et al., 2001).

In the auditory system, Galambos and colleagues (1981) reported a $40 \mathrm{~Hz}$ ( $\gamma$-band) oscillation from the human scalp, which was suggested to be generated by recurrent corticothalamic oscillation (Ribary et al., 1991) and was involved in novel stimulus processing (Haenschel et al., 2000) and in speech and non-speech

\footnotetext{
Received April 11, 2003; revised July 15, 2003; accepted July 16, 2003.

The work was supported by Hong Kong Research Grants Council Grant CERG PolyU5211/99M and by the Dean's Reserve of the University. I thank E. G. Jones for triggering discussions on the subject of this paper, L. M. Kitzes, P. W. F. Poon and B. Hu for critical reading of a previous version of this paper, C. L. H. Kam for data processing, and S. Q. Shi for anatomical assistance.

Correspondence should be addressed to Dr. Jufang He, Department of Rehabilitation Sciences, The Hong Kong Polytechnic University, Hung Hom, Kowloon, Hong Kong, China. E-mail: rsjufang@polyu.edu.hk.

Copyright $\odot 2003$ Society for Neuroscience $\quad$ 0270-6474/03/238281-10\$15.00/0
}

discrimination (Palva et al., 2002). High-frequency oscillation can be generated spontaneously in the cortex and evoked or induced by acoustic stimuli (Galambos et al., 1981; Barth and MacDonald, 1996; Brosch et al., 2002). They also can be modulated by thalamic stimulation (Barth and MacDonald, 1996; Brett and Barth, 1997). A $10 \mathrm{~Hz}(5-20 \mathrm{~Hz})$ tone-evoked oscillation that time-locked to tone onset was reported in the auditory cortex, the medial geniculate body (MGB), and the TRN (Shosaku and Sumitomo, 1983; Sally and Kelly, 1988; Eggermont, 1992; Bordi and LeDoux, 1994; Cotillon et al., 2000). The slow oscillation has been described in some dorsal thalamic nuclei, but not in the auditory thalamus (Steriade et al., 1993b,c; Hughes et al., 2002).

The phenomenon of a single neuron responding to a repeated stimulus with either no spike or a burst of discharges has been reported previously in the auditory thalamus and cortex (Suga and Horikawa, 1986; Hu et al., 1994; Hu, 1995; He and Hu, 2002). After a prolonged period of observation with an extracellular recording, we found a very slow rhythm of oscillation in spontaneous firings. An intracellular recording of thalamic neurons in an in vivo preparation enabled us to examine the neuronal membrane potential during acoustic stimulation, whereas the corticothalamic pathway was activated by electrical stimulation of the auditory cortex (Yan and Suga, 1996; He, 1997; Zhou and Jen, 2000). In the present study, we also investigated the repeated auditory response and the corticofugal interaction of the auditory thalamic neurons, in relation to the slow oscillation.

\section{Materials and Methods}

Animal preparation for extracellular recordings. Eighteen adult guinea pigs served as subjects of the present study. Of them, seven had also been used 
in a previous study to investigate the corticofugal modulation of ascending information on the thalamus (He et al., 2002; He, 2003), nine had been used in a study of OFF responsive properties (He, 2001, 2002), and two had been used for both studies. Anesthesia was induced and maintained with ketamine/xylazine ( 40 and $10 \mathrm{mg} / \mathrm{kg}$ initially, 10 and 2.5 $\mathrm{mg} / \mathrm{kg} / \mathrm{hr}$ thereafter, i.m.) during the surgical preparation and recording. The methodology has been described in detail previously ( $\mathrm{He}, 2001 ; \mathrm{He}$ et al., 2002). Briefly, the subject was mounted in a stereotaxic device after the induction of anesthesia. A midline incision was made in the scalp, and craniotomies were performed to enable us to map the auditory cortex and implant stimulation electrodes into it, and to access the MGB vertically in the left hemisphere (He, 1997, He et al., 2002). The head was fixed with two stainless steel bolts to an arm extended from the stereotaxic frame using acrylic resin. The right ear was then freed from the ear bar, so that the subject's head remained fixed to the stereotaxic device without movement.

Acoustic stimuli were generated digitally by a MALab system (Kaiser Instruments, Irvine, CA), which was controlled by a Macintosh computer (Semple and Kitzes, 1993; He, 1997). Acoustic stimuli were delivered to the subject via a dynamic earphone (Bayer DT-48; Bayer, Wuppertal, Germany) mounted in a probe. The sound pressure level (SPL) of the earphone was calibrated over a frequency range of $100 \mathrm{~Hz}$ to $35 \mathrm{kHz}$ under computer control using a condenser microphone ( 0.25 inch; Brüel and Kjær, Norcross, GA). The calibration was saved in the computer and used to compensate for the output intensity for each frequency (Semple and Kitzes, 1993). The subject was placed in a double-walled soundproof room (NAP Silentflo, Clayton, Australia). Repeated noise bursts and pure tones with a $5 \mathrm{msec}$ rise/fall time were used to examine the neuronal responses.

The MGB was accessed by a vertical approach with tungsten microelectrodes (9-12 M $\Omega$ ) in the stereotactically positioned subject, according to a guinea pig brain atlas (Rapisarda and Bacchelli, 1977). The vertical coordinate of the electrode was determined at a point slightly above the cortical surface at the first penetration, and was kept throughout the recording session. A single electrode was used for each experiment, so that not only the mediolateral and the rostrocaudal, but also the depth coordinates could be kept consistent over different penetrations during the experiment. In other words, a single coordinate system applied to all recording sites. This technique enabled us to reconstruct the physiological map of the whole frontal or sagittal auditory thalamic plane containing multiple penetrations, and to superimpose it with Nissl staining ( $\mathrm{He}$, 2001). We could record from 17 frontal planes and five parasagittal planes with an average of seven penetrations on each plane. Two planes each were recorded from five subjects, one plane each from 12 subjects, and only one penetration from one subject.

Animal preparation for intracellular recording. Ten guinea pigs served as subjects for intracellular recording. Anesthesia was initially induced with pentobarbital sodium (Nembutal $35 \mathrm{mg} / \mathrm{kg}$, i.p.; Abbott Labs, Irving, TX) and maintained by supplemental doses of the same anesthetic $(\sim 5-10 \mathrm{mg} / \mathrm{kg} / \mathrm{hr})$ during the surgical preparation and recording. The procedures for the head fixation and the craniotomy of the subject were the same as those for extracellular recording.

CSF was released through an opening on the back of the neck at the medulla level. Artificial respiration was applied to the animal, both sides of the animal's chest were opened, and its body was hung up to reduce vibrations of the brain caused by intrathoracic pressure.

We used a glass pipette as the recording electrode, filling it with $0.5 \mathrm{M}$ $\mathrm{KCl}$ ( $\mathrm{pH}$ 7.6, $0.05 \mathrm{~m}$ Tris $\mathrm{HCl}$ buffer). The resistance of the electrode was between 40 and $90 \mathrm{M} \Omega$. The electrode was advanced vertically from the top of the brain by the stepping motor. After the electrode was lowered to a depth of $4-5 \mathrm{~mm}$, the cortical exposure was sealed using low-meltingtemperature paraffin. When the electrode was near or in the targeting area, it was slowly advanced 1 or $2 \mu \mathrm{m}$ per step.

The electrode recorded a negative value in the membrane potential when it penetrated the membrane of a cell. After amplification, the membrane potential as well as the auditory stimulus and the electrical stimulation signals were stored on the computer with the aid of commercial software (AxoScope; Axon Instruments, Foster City, CA) (Fig. $1 A, B)$. In the present study, no current injections were made into the recorded neurons.

Electrical stimulation. The tonotopic organizations of the auditory cortex (the anterior and dorsocaudal auditory fields) were roughly mapped for extracellular recording over nine subjects to identify the electrical stimulation sites. To characterize the auditory cortex, we used $50 \mathrm{msec}$ tone pips ( $5 \mathrm{msec}$ rise/fall time, $>400 \mathrm{msec}$ interval) and most often recorded spikes from cell clusters rather than single cells. [The results have been published previously (He et al., 2002; He, 2003).] An electrode array consisting of three parallel electrodes was then implanted into the auditory cortex. We used electrical current pulse trains of $0.1 \mathrm{msec}$ width, $200 \mathrm{~Hz}$ frequency, and 30 pulses to activate the already mapped auditory cortex (Edeline et al., 1994; He, 1997; He et al., 2002). Electrical currents of 50-200 $\mu \mathrm{A}$ were applied to the auditory cortex, ipsilaterally to the recording thalamus through either a monopolar or bipolar lowimpedance electrode array. After a delay interval of $100 \mathrm{msec}$, a sound stimulus was delivered to the contralateral ear of the recording hemisphere after the end of the cortical stimulation (He, 1997, 2003).

Anatomical confirmation. Of 18 extracellular subjects, 12 were used for the purpose of anatomical confirmation. We failed in perfusions or terminated the experiments in the middle of the physiological study for the remaining six subjects. In the present study, we did not confirm the anatomical locations of recorded neurons with intracellular preparation.

For anatomical confirmation, a small lesion was made by passing a current of $1.0 \mu \mathrm{A}(20 \mathrm{sec})$ through the recording electrode $(9-12 \mathrm{M} \Omega)$ at the end of the last recording penetration. The subjects were deeply anesthetized with sodium pentobarbital and perfused transcardially with $0.9 \%$ saline followed by a mixture of $2.5 \%$ paraformaldehyde and $0.4 \%$ glutaraldehyde in $0.1 \mathrm{M}$ phosphate buffer, $\mathrm{pH}$ 7.3. The brains were dissected free and stored overnight in a $0.1 \mathrm{~m}$ phosphate buffer containing $30 \%$ sucrose. The thalami were cut transversely using a freezing microtome.

All sections of the 12 subjects were stained using the Nissl method. The sections were superimposed on the physiology map, using the electrode penetration tracks and the lesion for guidance. There was some shrinkage of the sections after the Nissl procedure. Enlargements of $10-13 \%$ of the Nissl images were made to match them to the physiology maps.

Statistical analysis. Because the oscillation in the present study was very slow, it required a long observation time. We set a long time window $(>80 \mathrm{sec})$ to monitor the neuronal activities in the present study. Raster displays were used to show the long-time oscillation of the neuronal spontaneous firing and their responses to auditory stimulus. We used $100 \mathrm{msec}$ noise bursts ( $5 \mathrm{msec}$ rise/fall time, $>400 \mathrm{msec}$ interval) as the stimulus in testing the auditory responses of the oscillation neurons. The temporal functions of the $\mathrm{ON}$ responses, as shown to the left of the raster display in Figure $2 \mathrm{~A}$, were drawn for all of the tested neurons. The ON function in Figure $2 \mathrm{~A}$ was obtained by counting all of the spikes in the first $100 \mathrm{msec}$ peristimulus time for each trial and drawn as a function of time. We also examined the temporal functions of the neuronal firing rate of the last $200 \mathrm{msec}$ (for those neurons with a stimulus interval $\geq 500$ $\mathrm{msec}$ ) or the last $100 \mathrm{msec}$ (for those neurons with a stimulus interval of $400 \mathrm{msec}$ ), as an example shown to the right of Figure $2 \mathrm{~A}$. The spikes occurring during the last $200 \mathrm{msec}$ of each trial were called after-stimulus activities.

The spectra of the oscillation frequencies were calculated from the neuronal firing rate over the long time course, using a fast-Fouriertransform (FFT) algorithm, as shown in the bottom panel of Figure $2 \mathrm{~A}$. Because the FFT algorithm required a data set with data points of the $n$th power of 2, we used either the first 64 or 128 data points in the present study.

The cross-correlation of the temporal functions of the ON responses and the after-stimulus activities was calculated over 14 neurons. An example was shown in Figure $2 B$, in which the cross-correlation was calculated over the lag time between the two temporal functions along the $y$-axis in Figure $2 A$.

The latency of the first spike of auditory response was calculated using MALab software. Because many slow-oscillation neurons often showed burst responses and no spikes to repeated auditory stimuli, we set a time 

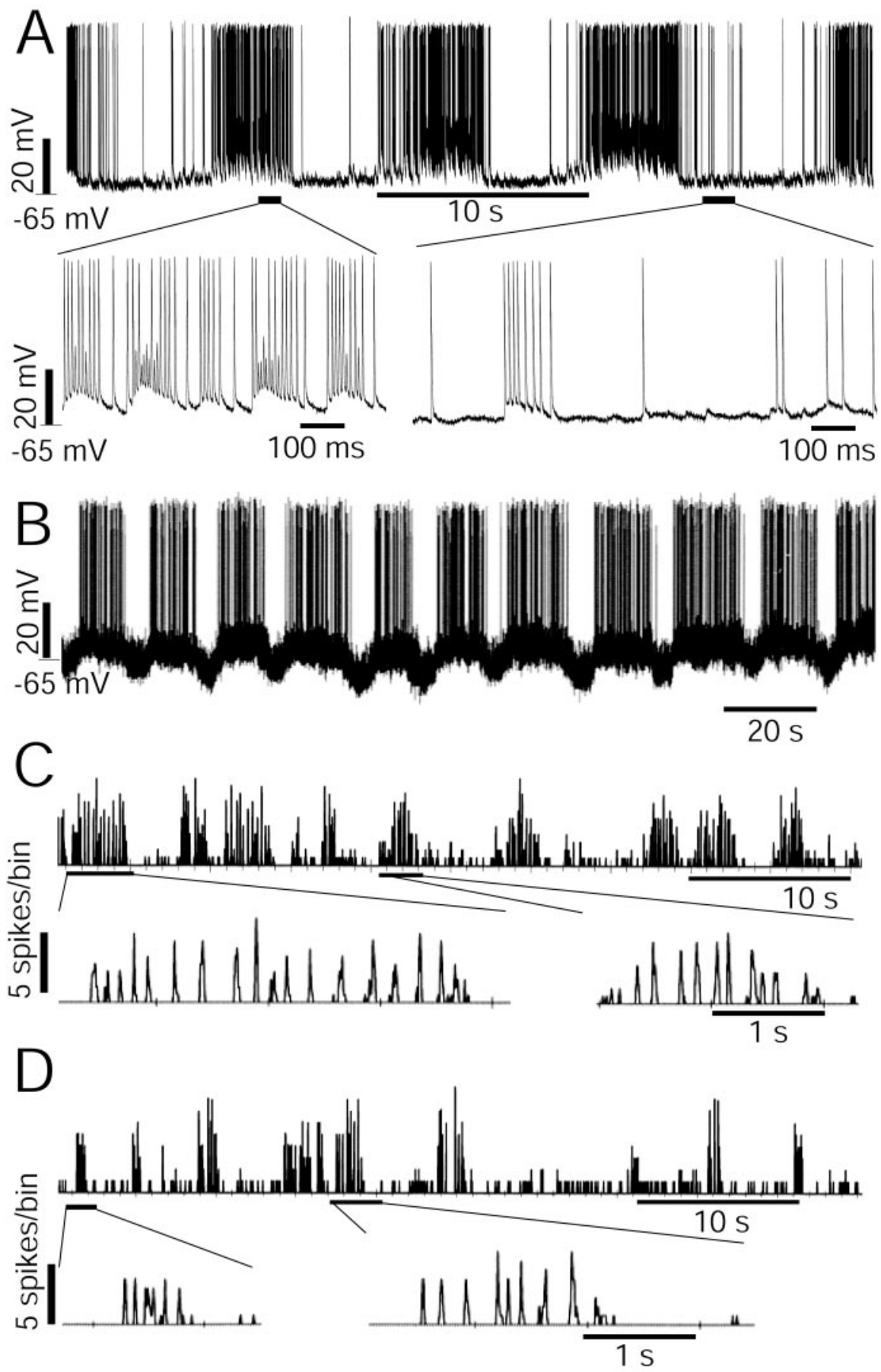

Figure 1. Slow oscillation of auditory thalamic neurons. $A, B$, Intracellular recordings show a slow membrane oscillation of two auditory thalamic neurons. $A$, The top row shows a rhythm of $0.11 \mathrm{~Hz}$ and the traces in the bottom row show stretched time traces at two phases in the oscillation rhythm. The membrane potential and the scale of the potential are shown to the left of the traces. $C, D$, Spontaneous firing rates of two neurons were recorded from the non-lemniscal MGB. Data were obtained through a MALab software package from extracellular recording. The firing rates were calculated over the spike sequences over $10 \mathrm{msec}$ bins. The top traces show the slow oscillation and the bottom traces show the expanded time axis and a faster rhythmic oscillation with burst firings. The scales of the firing rates are shown to the left of traces. The time scales are shown below the traces. The neurons in C and $D$ were located in the shell and in the rostromedial nuclei of the non-lemniscal MGB, respectively.

window of between 5 and $50 \mathrm{msec}$ to eliminate the error in detecting the first spike to each trial of the stimulus.

ANOVA was performed to examine whether there were differences among the latencies of oscillation neurons and the latencies of nonoscillation neurons in various divisions of the auditory thalamus. Post hoc tests of least significant difference were performed to detect the differ- ences between the mean latency of the oscillation neuron and each of the mean latencies of the responses in the various divisions. A paired Student's $t$ test was performed to compare the mean oscillation rhythms of the same group of neurons without corticofugal alteration and with corticofugal alteration. An unpaired Student's $t$ test was performed to examine the difference in oscillation frequencies between neurons in ketamine/xylazine-anesthetized animals and in pentobarbital sodium-anesthetized animals. The results were considered significant at the $95 \%$ confidence level $(p<0.05)$.

\section{Results}

Slow oscillation

We studied the slow oscillation of 122 auditory thalamic neurons, of which 62 were adopted in the analysis for the oscillation rhythm, being from 0.03 to $0.25 \mathrm{~Hz}$ (mean $\pm \mathrm{SD}, 0.11 \pm 0.05 \mathrm{~Hz}$ ). The neurons in Figure $1 A-D$ showed rhythms of $0.11,0.06,0.2$, and $0.22 \mathrm{~Hz}$, respectively. The intracellular recordings showed that a membrane oscillation is associated with the change in spike frequency (Fig. $1 A, B$ ). In the normal situation of the auditory thalamic neurons, when the resting membrane potential was between -55 and -70 $\mathrm{mV}$, the firing frequency was always positively associated with the membrane potential. When membrane was depolarized, neurons showed an increased spontaneous firing rate (Fig. 1) and an increased response to the auditory stimulus (Xiong et al., 2001). It was common to see another oscillation rhythm of $5-10 \mathrm{~Hz}$ on the peak phase of the slow oscillation wave (Fig. $1 A, C, D$, bottom rows).

Figures 2 and 3 show six more slowoscillation neurons. The raster display shows the neuronal responses to a noiseburst stimulus of 200 repeats and a 500 msec interstimulus interval. The slow oscillation can be seen only along the $y$-axis of the raster display. Fluctuations in spike frequency along the $x$-axis were beyond the slow rhythm. The temporal functions of the ON responses and the responses after a $300 \mathrm{msec}$ peristimulus time are shown to the left and right of the raster display. FFT analyses were carried on the temporal functions. The neuron in Figure 2 had an oscillation frequency of $0.11 \mathrm{~Hz}$. The neurons in Figure 3 had an oscillation frequency range between 0.1 and $0.16 \mathrm{~Hz}$. All of the neurons in Figures 2 and 3 responded to auditory stimulation.

The spectrum analyses of the oscillation neuron in Figure 2 are shown below the raster display. The response to the acoustic stimulus was either no spike or a burst of spikes. The rhythmic oscillation was obvious in the temporal functions for the ON responses and for the after-stimulus activities, shown on the left and right of the raster display, respectively. 

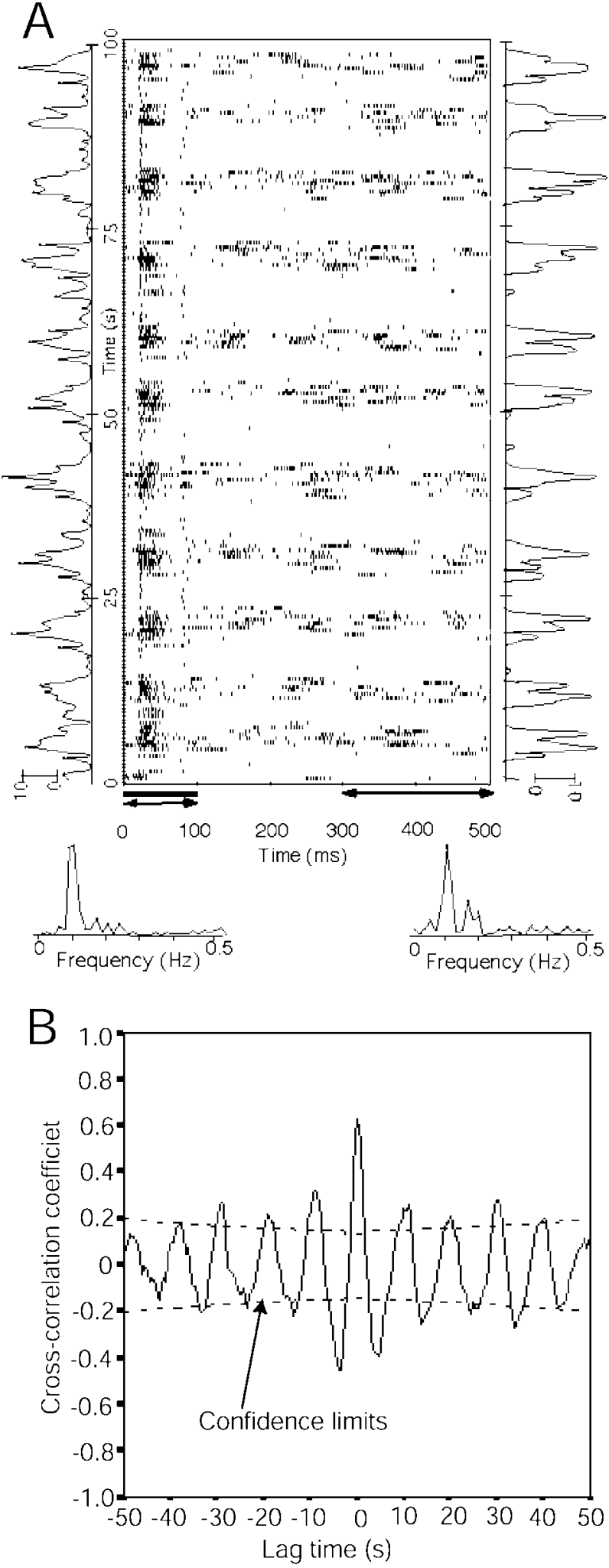

Figure 2. The extracellular recording of a slow-oscillation neuron. $A$, The raster display shows the neuronal response to a repeated noise-burst stimulus of $100 \mathrm{msec}$ duration, a 500 msec interval, and 200 trials. The raster display (i.e., the time of the occurrence of the spike relative to delivery of the stimulus) was stored in the computer used as the stimulus controller by the MALab software. The number of spikes of the $\mathrm{ON}$ responses acoustically evoked during each trial is shown as a function of the trial (temporal function) on the left of the raster display. The number of spikes occurring during the last $200 \mathrm{msec}$ of each trial (after-stimulus activities) is shown on the right. The time windows during which the spikes in the temporal functions were
The ON responses and after-stimulus activities of the oscillation neurons showed a good cross-correlation, with a mean crosscorrelation efficient, $r=0.55 \pm 0.13(n=14$, all with a confidence level of 95\%), as seen in Figure $2 B$, for which $r=0.66$.

\section{Response latency of the oscillation neurons}

We have randomly sampled 29 auditory responsive neurons with a clear slow oscillation, examples of which are shown in Figure $3 A$. Figure $3 A$, right, shows the raster displays of the first $50 \mathrm{msec}$ of the neuronal responses to auditory stimuli of the corresponding neurons. All of the neurons in the figure had a relatively longer latency than the average lemniscal neuron, which was $<10$ msec (He, 2002). The mean latency of the first spikes of the auditory responses calculated was $17.6 \pm 5.4 \mathrm{msec}(n=29$; range, 12.4-40.4 msec). The shortest latency was $>12 \mathrm{msec}$. The auditory response latency of the oscillation neurons was compared with that of the non-oscillation neurons in the lemniscal and non-lemniscal nuclei of the MGB, as shown in Figure $3 B$. The data on the non-oscillation neurons were sampled from data presented elsewhere (He, 2002). They were collected from the same group of subjects used in the present study.

The latency of the oscillation neurons showed a significant difference of $8.6 \mathrm{msec}$ ( $p<0.001$, ANOVA) with that of the neurons sampled from the central core of the lemniscal MGB (central MGv) and a significant difference of $4.5 \mathrm{msec}(p<0.01$, ANOVA) with that of the neurons from the border region of the MGv. However, the difference of the latencies of the oscillation neurons and of the non-oscillation neurons sampled from the non-lemniscal MGB was very small and statistically not significant $(0.3 \mathrm{msec}, p=0.811)$.

\section{Location of the oscillation neurons}

As mentioned in Materials and Methods, the extracellular recordings were obtained from the experimental subjects, the results for other purposes of which have been published previously (He, 2001, 2002, 2003; He and Hu, 2002; He et al., 2002). Neurons recorded from the 12 subjects could be localized with anatomical confirmation of multiple penetration lesions and/or electrical lesions. Neurons from two subjects, for which physiological results were carefully mapped over 13 and 24 penetrations, were estimated based on the physiological maps (one of which is shown in Fig. 4 of He, 2001).

Of a subset of the oscillatory neurons $(n=106)$, whose locations could be examined using their physiological map and/or anatomical map, 29 were located in the shell nucleus (MGs), 23 in the caudomedial nucleus $(\mathrm{MGcm}), 15$ in the rostromedial nucleus (MGrm), 23 in the dorsal nucleus (MGd), and 16 on the border between the ventral nucleus (MGv) and other nuclei. No oscillation neurons were found in the central region of the MGv. Figure 4 shows the spatial locations of the oscillation neurons in three subjects. In summary, all of the oscillation neurons were located in the non-lemniscal nuclei or on the border regions between the lemniscal and non-lemniscal nuclei of the MGB. The raster display shown in Figure $4 B$ was an ON-OFF neuron, which typically appeared on the border of the lemniscal and non-

counted are shown with arrowheads below the raster display. The spectral analyses of the $\mathrm{ON}$ responses and the after-stimulus activities are shown below the raster display. The spectra were calculated with an FFT algorithm over the temporal functions. The acoustic stimulus was a repeated noise burst $100 \mathrm{msec}$ in duration and an SPL $60 \mathrm{~dB}$ in intensity. The neuron was located in the shell nucleus of the non-lemniscal MGB. B, Cross-correlation between the temporal function of the $0 \mathrm{~N}$ response and that of the after-stimulus activities. 

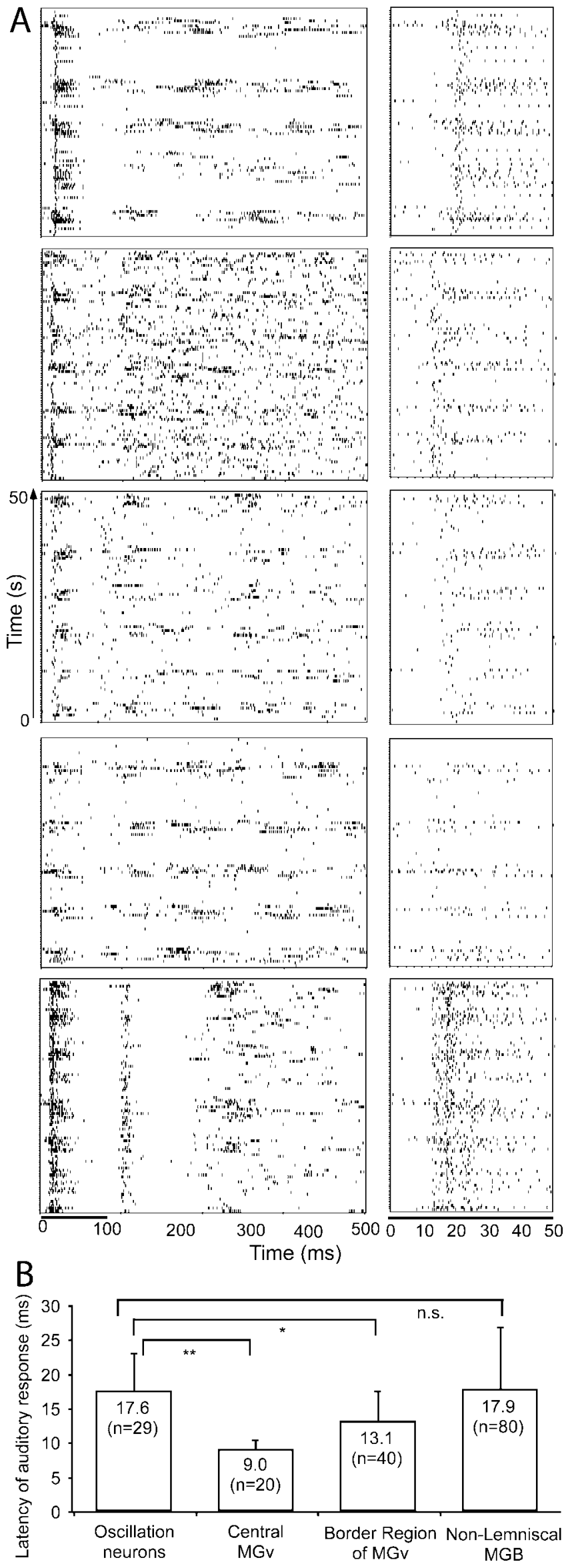

lemniscal nuclei, or in the non-lemniscal nuclei (He, 2001, 2002). The neuron in the lowest row of Figure $3 A$ was also an ON-OFF neuron. Some oscillation neurons showed a very weak auditory response, as seen in Figure $3 A$, fourth row.

\section{Corticofugal alteration of the thalamic oscillation}

The slow oscillation of the thalamic neuron can be altered by the activated corticofugal pathway, as seen in the examples shown in Figure $5 A$ and $B$. Electrical stimulation of the primary auditory cortex produced slow EPSPs or slow IPSPs in some thalamic neurons or TRN neurons (Fig. 5A). The slow EPSPs depolarized the oscillating neuron while it was in the hyperpolarized phase (Fig. 5A, bottom). Of 17 neurons examined, the oscillations of seven were disrupted (Fig. 5A) and the oscillations of eight were altered, whereas the auditory cortex was stimulated with trains of stimulus pulses. Of these eight neurons, the frequencies of oscillation of six increased (Fig. 5B) and two decreased. Their average rhythm changed from $0.07 \pm 0.03$ to $0.11 \pm 0.04 \mathrm{~Hz}(n=8, p=$ $0.066, t$ test) after stimulation of the auditory cortex.

\section{Repeated acoustic stimulus induces a better oscillation rhythm}

Acoustic stimulation can also modulate the frequency of oscillation. All four of the neurons examined showed such changes, with a more regular rhythm, after repetitive acoustic stimulation. An example is shown in Figure 6. The spontaneous firing of the neuron (Fig. 6, left) shows an oscillation in the raster display, whereas the rhythm became clearer in the Figure 6, right, where a repetitive acoustic stimulus was applied. It is not difficult to identify a better oscillation rhythm in Figure 6, right, than in Figure 6, left, in the time course of the spike-number traces for the last 100 msec of the stimulus interval, shown on the right of each raster display. Spectral analyses of the spike-number traces as indicated in Figure 6 revealed a narrower peak after the induction of the acoustic stimulus, suggesting a more regular rhythm. However, its main rhythm $(0.20 \mathrm{~Hz})$ did not seem to change.

\section{Anesthetic effect on the oscillation rhythm}

There was a significant frequency difference $(0.058 \mathrm{~Hz}, p<0.001$, $t$ test) between neurons in the ketamine/xylazine-anesthetized animals $(0.122 \pm 0.046 \mathrm{~Hz}, n=49$ neurons $)$ and in the pentobarbital sodium-anesthetized animals $(0.065 \pm 0.024 \mathrm{~Hz} ; n=13$ neurons).

Tone-evoked $\beta$ - and $\gamma$-oscillation lasts for a period of $\sim 1 \mathrm{sec}$ (Cotillon et al., 2000; Haenschel et al., 2000). However, the slow oscillation of $0.03-0.25 \mathrm{~Hz}$ was observed in the present study to have lasted for over $13 \mathrm{~min}$.

Figure 3. Auditory responses and the mean latency of thalamic oscillation neurons. $A$, The raster displays of 50 sec each of five neurons in the left panel were sampled from their responses to a repeated noise-burst stimulus. The right panel shows the auditory responses of the first 50 msec for each corresponding neuron in the left panel. The duration of the noise burst shown under the lowest raster display applies to all the neurons above. The time scale shown below the lowest raster displays and on the left likewise applies to all. The neurons in up- down order were sampled from the caudomedial, caudomedial, shell, shell, and rostromedial nuclei of the non-lemniscal MGB, respectively. $B$, Mean latency of the first spikes of 29 oscillation neurons compared with the mean latency of the non-oscillation neurons in the lemniscal and nonlemniscal MGB. The data of the non-oscillation neurons were sampled from data presented previously (He, 2002). They were collected from the same group of subjects. The numbers in the bars show the mean latencies. $n$, Sampling size. ${ }^{*} p<0.01$ (ANOVA); ${ }^{* *} p<0.001$; n.s., not significant $(p=0.811)$. 


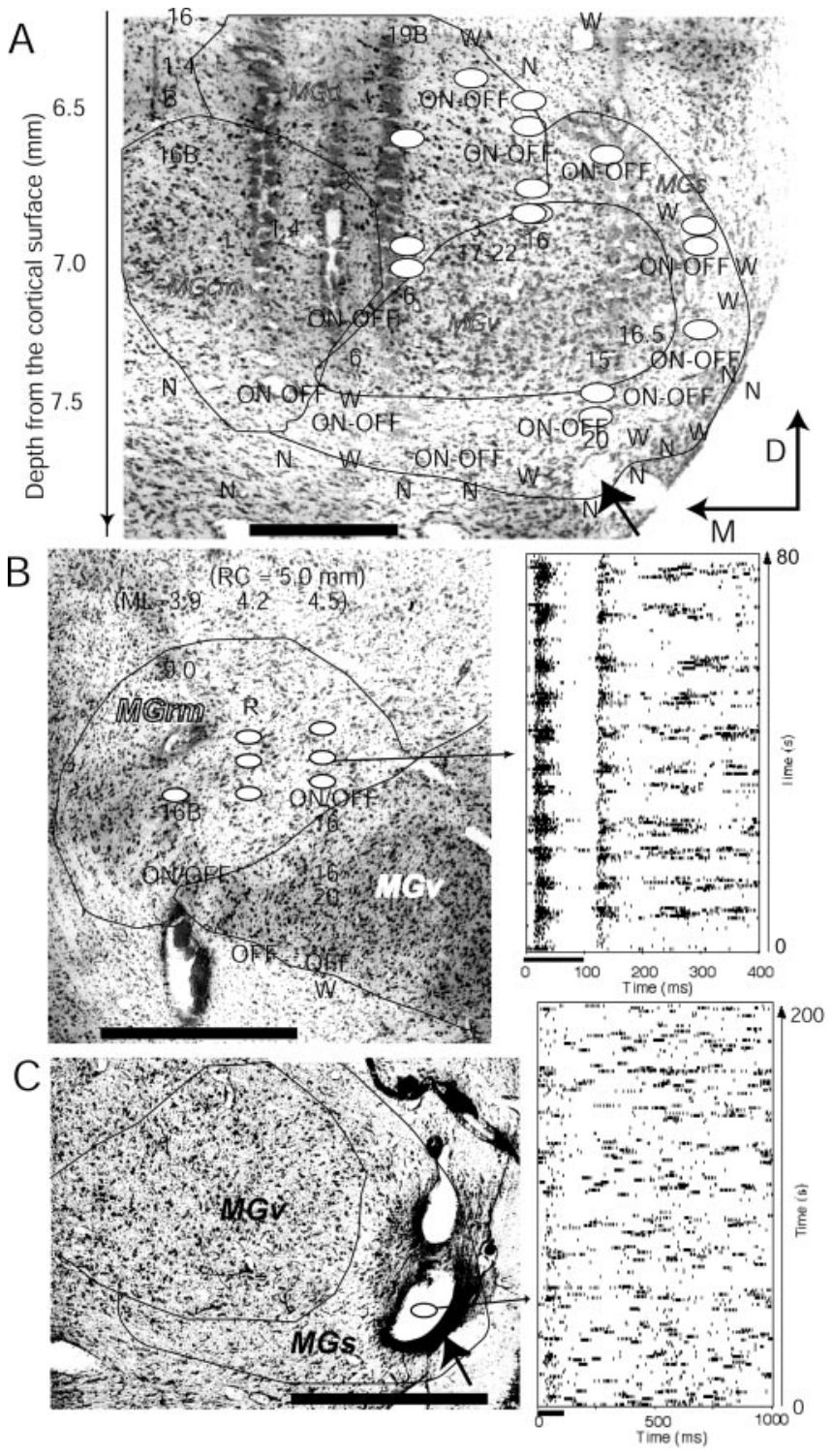

Figure 4. Spatial locations of the oscillation neurons in the auditory thalamus. A-C, Maps of multiple penetrations of physiological recordings were superimposed with the Nissl staining of frontal sections. Ovals indicate the locations of oscillation neurons. Electrode tracks and the lesions were used as landmarks to match the physiological map with the Nissl staining. In $A$ and $C$, the lesion indicated with the arrowheads was made at the last recording penetration. Fourteen penetrations were made on the same frontal plane shown in $A$. Because the subject was also used for other purposes and the method has been described previously (Fig. 3 of He, 2001), we superimposed only the minimum amount of physiological information onto the Niss section here. In $B$, three penetrations were made on the frontal plane (partial results were presented in Fig. $5 B$ of He et al., 2002). In C, the lesion was made after we recorded an oscillation neuron in the first penetration. Only one penetration was made on this subject. A raster display of the auditory response of an oscillation neuron to a repetitive auditory stimulus was sampled from each of subjects $B$ and $C$ and shown on the right side of the maps. The auditory stimulus for the raster display in $B$ and $C$ was a noise burst of 100 msec duration and $60 \mathrm{~dB}$ SPL intensity. Numbers represent the best frequencies. W, Weak auditory response; $N$, no response; $B$ after a number, broad tuning; OFF, OFF response; ON-OFF, ON-OFF response; ML, mediolateral; RC, rostrocaudal; $M$, medial; $D$, dorsal. Scale bar, $0.5 \mathrm{~mm}$.

\section{Discussion}

Slow oscillation

The slow oscillation frequencies of between 0.1 and $1 \mathrm{~Hz}$ (mostly between 0.5 and $0.9 \mathrm{~Hz}$ ) that have been reported previously (Steriade et al., 1993a-d) are related to slow-wave sleep and are also affected by anesthetic. A lower rhythm has been reported for
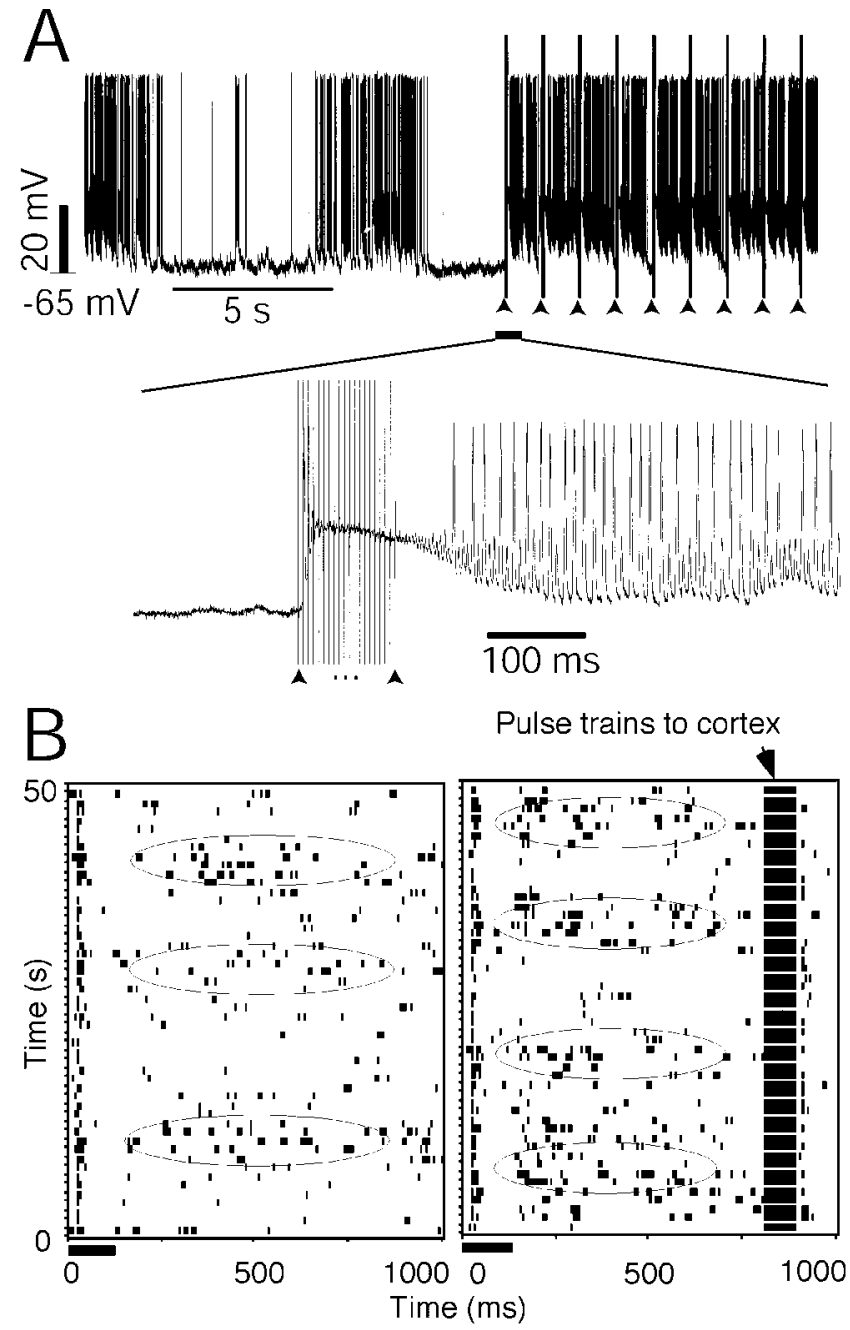

Figure 5. Thalamic oscillation altered by the corticofugal modulation. $A$, An intracellular recording shows that the thalamic oscillation was terminated, whereas the electrical stimulation of the auditory cortex was turned on. The bottom panel shows the time-axis expanded from when electrical pulses were applied to stimulate the cortex. Arrowheads indicate the pulse trains of the electrical currents applied to the auditory cortex. The electrical stimulation current was $200 \mu \mathrm{A}$. B, Raster displays show the neuronal responses to a repeated auditory stimulus. The neuron was located in the caudomedial nucleus of the non-lemniscal MGB. Left, Only auditory stimulus was given to the subject; right, the auditory cortex was activated $100 \mathrm{msec}$ before the auditory stimulus was delivered to the subject. The rhythm became faster when the cortex was stimulated. The ellipses indicate the peak phases of the slow oscillation waves. The electrical stimulation current was $100 \mu \mathrm{A}$.

urethane anesthesia compared with ketamine (Steriade et al., 1993b). In the present study, the oscillation frequency was even lower, from 0.03 to $0.25 \mathrm{~Hz}$; it was also dependent on the anesthetic. The frequency slowed down when we shifted the anesthetic from ketamine/xylazine to sodium pentobarbital. The oscillations were then believed to be slow-wave oscillations.

In the auditory system, $\gamma(20-80 \mathrm{~Hz})$ and $\beta(5-20 \mathrm{~Hz})$ oscillations have been reported and could be either spontaneously generated or auditorily evoked/induced (Galambos et al., 1981; Shosaku and Sumitomo, 1983; Sally and Kelly, 1988; Ribary et al., 1991; Eggermont, 1992; Bordi and LeDoux, 1994; Barth and MacDonald, 1996; Cotillon et al., 2000; Brosch et al., 2002; Palva et al., 2002; for detailed definitions of varied oscillations, see Castro-Alamancos and Connors, 1997). Recently, CotillonWilliams and Edeline (2003) observed a stimulus-evoked oscillation of 5-15 Hz in an anesthetized rat and a non-stimulus-locked 


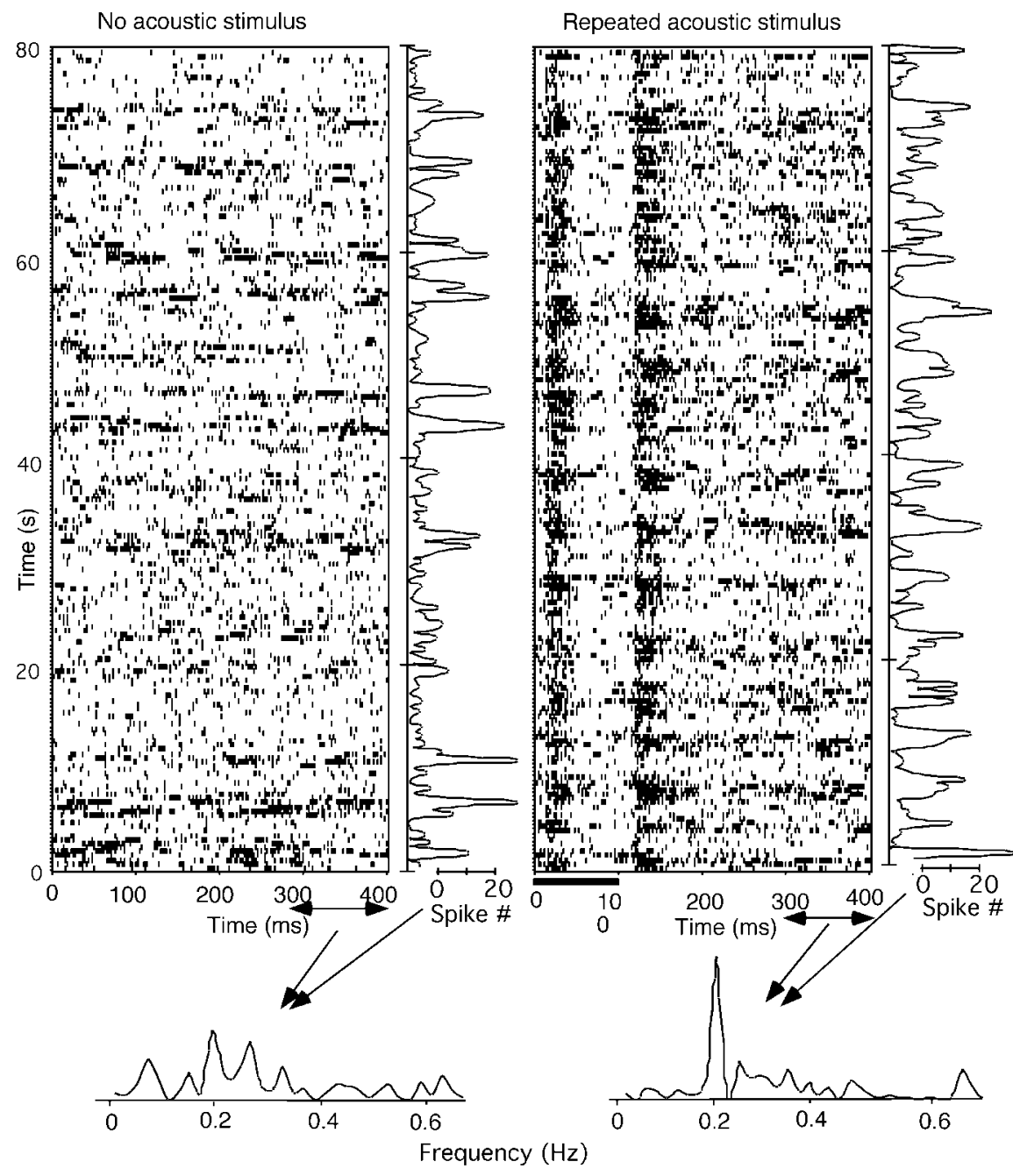

Figure 6. The rhythm of the thalamic oscillation improved by a repeated auditory stimulus. Top, Raster displays of the spontaneous firing (left) and the responses to repeated acoustic stimulus of a noise burst of 100 msec duration and $60 \mathrm{~dB} \mathrm{SPL}$ intensity (right). The time courses of the firing rate of the last $100 \mathrm{msec}$ duration (spike-trial function), as indicated below the raster displays, are shown to the right of the displays. The lower panel shows the frequency analyses of the spike-trial functions for both conditions. The neuron was located in the rostromedial nucleus of the non-lemniscal MGB.

oscillation in a slow-wave-sleep rat, but no oscillations in waking and paradoxical sleep animals. Together with the present finding and that of Steriade and colleagues (1993b), the results suggest that the frequency of oscillation is anesthesia- and statedependent. Slow oscillation characterizes slow-wave sleep and fast rhythms occur during waking and REM sleep (Steriade and Timofeev, 2003). However, this is the first report of a slow oscillation in the auditory system.

The slow oscillation is believed to be generated in the neocortical network (Steriade et al., 1993d,e). The thalamus not only participates in the slow oscillation, but also actively shapes the sleeping oscillation (Steriade et al., 1993b, c; Contreras and Steriade, 1995; Contreras et al., 1996). A recent in vitro study by Hughes and colleagues (2002) showed that a slow oscillation can be obtained intrinsically from the thalamocortical neurons by activating the metabotropic glutamate receptors. The activation of the corticothalamic pathway can modulate thalamic firing through metabotropic glutamate receptors (McCormick and von Krosigk, 1992). The observation that cortical neurons continue to generate sustained periods of action potentials during sleep supports the view that the metabotropic glutamate receptors are substantially activated (Steriade et al., 2001; Hughes et al., 2002). The present study has shown that the thalamic slow oscillation can be terminated by cortical activation. Combined with the in vitro and in vivo results, we may speculate that the cortex initiates the slow oscillation, the thalamus participates and further develops it to a global attribute (Contreras and Steriade, 1995; Contreras et al., 1996; Hughes et al., 2002), and the cortex terminates it.

The TRN neurons are supposedly involved in the tone-evoked oscillation (Cotillon et al., 2000) and in slow and spindlelike oscillations (Steriade et al., 1993b,c; Contreras et al., 1996; Grenier et al., 1998; Golshani and Jones, 1999; Steriade, 2001a). In a slice experiment of $\mathrm{GABA}_{\mathrm{A}}$ receptor $\beta_{3}$ knock-out mice, Huntsman and colleagues (1999) found that the oscillatory synchrony had dramatically intensified, suggesting that the reticular nucleus is very much involved in the regulation of the oscillation.

The peak amplitude of corticothalamic EPSCs is $\sim 2.5$ times higher in the TRN neurons, compared with thalamocortical neurons (Golshani et al., 2001; Liu et al., 2001). This indicates that the corticofugal fibers to the TRN neurons have a stronger control over the excitability of the TRN neurons (Steriade, 2001b). The TRN neurons extend dendrites within the thin reticular sheet, which enable them to receive projections from a wide cortical region, and project to widespread areas in the ventroposterior nucleus of the thalamus (Liu et al., 1995). These, together with the results of other studies, lead to the reasonable conclusion that the control of the thalamus via the TRN is widespread (Jones, 1975, 1985; Ohara et al., 1980; Yen et al., 1985; Liu et al., 1995; Bourassa et al., 1995; Cox et al., 1997; Crabtree, 1998; Crabtree and Isaac, 2002).

\section{Oscillation in the non-lemniscal MGB}

A major finding of the present study was the localization of the oscillation neurons in the auditory thalamus. All of the oscillation neurons whose locations could be confirmed anatomically were located in the non-lemniscal MGBs or on the border region of the lemniscal and non-lemniscal MGBs.

All randomly sampled, oscillation neurons had a long latency of auditory response $(>12.4 \mathrm{msec})$. The mean latency of the oscillation neurons was significantly longer than that of the nonoscillation neurons in the lemniscal MGB and similar to that of the non-oscillation neurons in the non-lemniscal MGB. It has been well documented that non-lemniscal MGBs show a greater latency than lemniscal MGBs (Calford, 1983). The result of the longer responsive latency of the oscillation neurons would agree with the anatomical location in the non-lemniscal MGBs.

Some oscillatory auditory neurons showed burst-like patterns (Figs. 2, 3, and 4). Burst-firing patterns can be observed in both lemniscal and non-lemniscal MGBs but are more often seen in 
the non-lemniscal nuclei and on the border region between the lemniscal and non-lemniscal nuclei of the MGB ( $\mathrm{Hu}, 1995$; Mooney et al., 1995; $\mathrm{He}$ and $\mathrm{Hu}, 2002$ ). The present result explains the previous observation that these neurons may show either no spike or a burst of spikes in response to auditory stimuli (Suga and Horikawa, 1986; Hu et al., 1994; $\mathrm{Hu}, 1995 ; \mathrm{He}$ and $\mathrm{Hu}$, 2002).

Because OFF/ON-OFF neurons are located only in nonlemniscal MGBs and the border regions between the lemniscal and non-lemniscal MGBs (He, 2001), that some oscillation neurons in the present study showed an ON-OFF response (Figs. 3, 4, and 6) would strengthen our finding that the oscillation neurons are located in non-lemniscal MGBs and the border regions between the lemniscal and non-lemniscal MGBs.

It has been suggested that the parallel pathways via the lemniscal and non-lemniscal nuclei of the MGB are functionally segregated. The non-lemniscal MGB neurons show long latency, burst firing, broad/non-frequency-tuning, non-tonotopic organization, OFF responses, interaction with the limbic system, and multimodal responses (de Ribaupierre and Toros, 1976; Andersen et al., 1980; Calford 1983; Calford and Aitkin, 1983; Imig and Morel, 1983, 1984, 1985; Hu, 1995; Kosaki et al., 1997; He et al., 1997; Rauschecker et al., 1997; He and Hashikawa, 1998; $\mathrm{He}, 2001$; He and $\mathrm{Hu}, 2002$ ). Our recent study showed that cortical activation switched off the $\mathrm{ON}$ responses of the nonlemniscal MGB neurons and facilitated the responses of lemniscal MGB neurons in the guinea pig (He et al., 2002; He, 2003).

The frequency of the second rhythm in Figure 1 is similar to that of the tone-evoked $10 \mathrm{~Hz}$ oscillation in the auditory cortex, MGB, and TRN (Shosaku and Sumitomo, 1983; Sally and Kelly, 1988; Eggermont, 1992; Bordi and LeDoux, 1994; Cotillon et al., $2000)$, and to that of the spindle oscillation (7-14 Hz) found in other parts of the thalamus (Hu et al., 1989a,b; Huguenard and Prince, 1992; Contreras and Steriade, 1996; Terman et al., 1996). Spindle oscillation, which is believed to be generated at the thalamic level, can be triggered and controlled by cortical and brainstem stimulations (Mulle et al., 1986; Hu et al., 1989a; Huguenard and Prince, 1992, 1994; Contreras and Steriade, 1996; Terman et al., 1996; Blumenfeld and McCormick, 2000). However, the mechanism of the second rhythm needs additional investigation.

\section{Possible functional implications}

Although no major differences in intrinsic membrane features or synaptic responses between the lemniscal and non-lemniscal nuclei of the MGB were observed in in vitro slice preparations (Bartlett and Smith, 1999), the dorsal division of the non-lemniscal MGB receives many GABAergic giant axon terminals, whereas the lemniscal MGB is void of those terminals (Huang et al., 1999; Winer et al., 1999). The giant GABAergic axons are probably from the RTN neurons (He, 2003). In comparison, the lemniscal MGB receives a much stronger direct corticothalamic projection than does the non-lemniscal MGB (Winer and Larue, 1987).

The medial division (MGm) of the non-lemniscal MGB projects to all auditory fields and other sensory cortical fields, whereas the lemniscal nucleus projects only to the primary auditory field (Winer et al., 1977; Andersen et al., 1980; Winer and Morest, 1983; Imig and Morel, 1983, 1984). The MGm also projects to and receives input from the amygdala and the basal ganglia (Wepsic and Sutin, 1964; LeDoux et al., 1990; Cruikshank et al., 1992; Shinonaga et al., 1994). The cortical projection of the lemniscal MGB is mainly to cortical layers IV and IIIb, whereas the MGm projects primarily to layer I of the auditory cortex (Sousa-Pinto, 1973; Jones and Burton, 1976; Mitani et al., 1984;
Niimi et al., 1984; Winer, 1992; Hashikawa et al., 1995). Layer I consists of mainly neuropil development and few neurons (Winer, 1992). Of the neurons, $>90 \%$ are GABAergic, and no pyramidal neurons are found (Winer and Larue, 1989; Prieto et al., 1994). Many dendritic profiles in layer I are from the pyramidal neurons in layers II-V, suggesting that possible direct facilitation and/or indirect inhibition (via the layer I GABAergic neurons) from layer I may reach most of the other layers of the cortex (Winer, 1992). The MGm consists of large and deeply Nisslstained cells that project diffusely to layer I of the entire auditory cortex, hinting at a power to adjust the total activity of the auditory cortex (Winer and Morest, 1983; Redies et al., 1989; He, 2003).

The present results suggest one more function of the nonlemniscal MGB, the involvement of the global alertness of the auditory cortex or an even wider region of the brain, because the slow oscillation was associated with drowsiness and slow-wave sleep (Steriade et al., 1993a). The in vivo intracellular recording of the slow-oscillation neurons of the present study showed that the oscillation could be terminated by cortical stimulation. We also confirmed that positive interactions of the corticofugal pathway could either terminate or accelerate the oscillation. In conclusion, only the non-lemniscal MGB is involved in the slow thalamocortical oscillation and can thus control the global alertness of the auditory and related cortices.

\section{References}

Andersen RA, Knight PL, Merzenich MM (1980) The thalamocortical and corticothalamic connections of AI, AII, and the anterior auditory field (AAF) in the cat: evidence for two largely segregated systems of connections. J Comp Neurol 194:663-701.

Bal T, Debay D, Destexhe A (2000) Cortical feedback controls the frequency and synchrony of oscillations in the visual thalamus. J Neurosci 20:7478-7488.

Barth DS, MacDonald KD (1996) Thalamic modulation of high-frequency oscillating potentials in auditory cortex. Nature (Lond) 383:78-81.

Bartlett EL, Smith PH (1999) Anatomic, intrinsic, and synaptic properties of dorsal and ventral division neurons in rat medial geniculate body. J Neurophysiol 81:1999-2016.

Blumenfeld H, McCormick DA (2000) Corticothalamic inputs control the pattern of activity generated in thalamocortical networks. J Neurosci 20:5153-6162.

Bordi F, LeDoux J (1994) Response properties of single units in areas of rat auditory thalamus that project to the amygdale. I. Acoustic discharge patterns and frequency receptive fields. Exp Brain Res 98:261-274.

Bourassa J, Pinault D, Deschênes M (1995) Corticothalamic projections from the cortical barrel field to the somatosensory thalamus in rats: a single-fibre study using biocytin as an anterograde tracer. Eur J Neurosci 7:19-30.

Brett B, Barth D (1997) Subcortical modulation of high-frequency (gamma band) oscillating potentials in auditory cortex. J Neurophysiol 78:573-581.

Brosch M, Budinger E, Scheich H (2002) Stimulus-related gamma oscillations in primate auditory cortex. J Neurophysiol 87:2715-2725.

Calford MB (1983) The parcellation of the medial geniculate body of the cat defined by the auditory response properties of single units. J Neurosci 3:2350-2364.

Calford MB, Aitkin LM (1983) Ascending projections to the medial geniculate body of the cat: Evidence for multiple, parallel auditory pathways through thalamus. J Neurosci 3:2365-2380.

Castro-Alamancos MA, Connors BW (1997) Thalamocortical synapses. Prog Neurosci:581-606.

Contreras D, Destexhe A, Sejnowski TJ, Steriade M (1996) Control of spatiotemporal coherence of a thalamic oscillation by corticothalamic feedback. Science 274:771-774.

Contreras D, Steriade M (1995) Cellular basis of EEG slow rhythms: a study of dynamic corticothalamic relationships. J Neurosci 15:604-622.

Contreras D, Steriade M (1996) Spindle oscillation in cats: the role of corti- 
cothalamic feedback in a thalamically generated rhythm. J Physiol (Lond) 490:159-179.

Cotillon N, Nafati M, Edeline J-M (2000) Characteristics of reliable toneevoked oscillations in the rat thalamo-cortical auditory system. Hear Res 142:113-130.

Cotillon-Williams N, Edeline J-M (2003) Evoked oscillations in the thalamo-cortical auditory system are present in anesthetized but not in unanesthetized rats. J Neurophysiol 89:1968-1984.

Cox CL, Huguenard JR, Price DA (1997) Nucleus reticularis neurons mediate diverse inhibitory effects in thalamus. Proc Natl Acad Sci USA 94:8854-8859.

Crabtree JW (1998) Organization in the auditory sector of the cat's thalamic reticular nucleus. J Comp Neurol 390:167-182.

Crabtree JW, Isaac JTR (2002) New intrathalamic pathways allowing modality-related and cross-modality switching in the dorsal thalamus. J Neurosci 22:8754-8761.

Cruikshank SJ, Edeline JM, Weinberger NM (1992) Stimulation at a site of auditory-somatosensory convergence in the medial geniculate nucleus is an effective unconditioned stimulus for fear conditioning. Behav Neurosci 106:471-483.

de Ribaupierre F, Toros A (1976) Single unit properties related to the laminar structure of the MGN. Exp Brain Res Suppl 1:503.

Destexhe A, Babloyantz A, Sejnowski TJ (1993) Ionic mechanisms for intrinsic slow oscillations in thalamic relay neurons. Biophys J 65:1538-1552.

Edeline JM, Hars B, Maho C, Hennevin E (1994) Transient and prolonged facilitation of tone-evoked responses induced by basal forebrain stimulations in the rat auditory cortex. Exp Brain Res 97:373-386.

Eggermont JJ (1992) Stimulus induced and spontaneous rhythmic firing of single units in cat primary auditory cortex. Hear Res 61:1-11.

Galambos R, Makeig S, Talmachoff PJ (1981) A $40 \mathrm{~Hz}$ auditory potential recorded from the human scalp. Proc Natl Acad Sci USA 78:2643-2647.

Golshani P, Jones EG (1999) Synchronized paroxysmal activity in the developing thalamocortical network mediated by corticothalamic projections and "silent" synapses. J Neurosci 19:2865-2875.

Golshani P, Liu X-B, Jones EG (2001) Differences in quantal amplitude reflect GluR4-subunit number at corticothalamic synapses on two populations of thalamic neurons. Proc Natl Acad Sci USA 98:4172-4177.

Grenier F, Timofeev I, Steriade M (1998) Leading role of thalamic over cortical neurons during postinhibitory rebound excitation. Proc Natl Acad Sci USA 95:13929-13934.

Haenschel C, Baldeweg T, Croft RJ, Whittington M, Gruzelier J (2000) Gamma and beta frequency oscillations in response to novel auditory stimuli: a comparison of human electroencephalogram (EEG) data with in vitro models. Proc Natl Acad Sci USA 97:7645-7650.

Hashikawa T, Molinari M, Rausell E, Jones EG (1995) Patchy and laminar terminations of medial geniculate axons in monkey auditory cortex. J Comp Neurol 362:195-208.

He J (1997) Modulatory effects of regional cortical activation on the onset responses of the cat medial geniculate neurons. J Neurophysiol 77:896-908.

He J (2001) ON and OFF pathways segregated at the auditory thalamus of the guinea pig. J Neurosci 21:8672-8679.

He J (2002) OFF responses in the auditory thalamus of the guinea pig. J Neurophysiol 88:2377-2386.

He J (2003) Corticofugal modulation on both ON and OFF responses in the non-lemniscal auditory thalamus of the guinea pig. J Neurophysiol 89:367-381.

He J, Hashikawa T (1998) Connections of the dorsal zone of cat auditory cortex. J Comp Neurol 400:334-348.

He J, Hu B (2002) Differential distribution of burst and single-spike responses in auditory thalamus. J Neurophysiol 88:2152-2156.

He J, Hashikawa T, Ojima H, Kinouchi Y (1997) Temporal integration and duration tuning in the dorsal zone of cat auditory cortex. J Neurosci 17:2615-2625.

He J, Yu YQ, Xiong Y, Hashikawa T, Chan YS (2002) Modulatory effect of cortical activation on the lemniscal auditory thalamus of the guinea pig. J Neurophysiol 88:1040-1050.

Hu B (1995) Cellular basis of temporal synaptic signalling: an in vitro electrophysiological study in rat auditory thalamus, J Physiol (Lond) 483:167-182.

Hu B, Senatorav V, Mooney D (1994) Lemniscal and non-lemniscal synaptic transmission in rat auditory thalamus. J Physiol (Lond) 479:217-231.
Hu B, Steriade M, Deschênes M (1989a) The effects of brainstem peribrachial stimulation on perigeniculate neurons: the blockage of spindle waves. Neuroscience 31:1-12.

Hu B, Steriade M, Deschênes M (1989b) The cellular mechanism of thalamic ponto-geniculo-occipital waves. Neuroscience 31:25-35.

Huguenard JR, Prince DA (1992) A novel T-type current underlies prolonged $\mathrm{Ca}^{2+}$-dependent burst firing in GABAergic neurons of rat thalamic reticular neurons. J Neurosci 12:3804-3817.

Huguenard JR, Prince DA (1994) Intrathalamic rhythmicity studied in vitro: nominal $\mathrm{T}$-current modulation causes robust anti-oscillatory effects. J Neurosci 14:5485-5502.

Huang CL, Larue DT, Winer JA (1999) GABAergic organization of the cat medial geniculate body. J Comp Neurol 415:368-392.

Hughes SW, Cope DW, Blethyn KL, Crunelli V (2002) Cellular mechanisms of the slow $(<1 \mathrm{~Hz})$ oscillation in thalamocortical neurons in vitro. Neuron 33:947-958.

Huntsman MM, Porcello DM, Homanics GE, DeLorey TM, Huguenard JR (1999) Reciprocal inhibitory connections and network synchrony in the mammalian thalamus. Science 283:541-543.

Imig TJ, Morel A (1983) Organization of the thalamocortical auditory system in the cat. Annu Rev Neurosci 6:95-120.

Imig TJ, Morel A (1984) Topographic and cytoarchitectonic organization of thalamic neurons related to their targets in low-, middle- and highfrequency representations in cat auditory cortex. J Comp Neurol 227:511-539.

Imig TJ, Morel A (1985) Tonotopic organization in ventral nucleus of medial geniculate body in the cat. J Neurophysiol 53:309-340.

Jones EG (1975) Some aspects of the organization of the thalamic reticular complex. J Comp Neurol 162:285-308.

Jones EG (1985) The thalamus. New York: Plenum.

Jones EG, Burton H (1976) Areal differences in the laminar distribution of thalamic afferents in cortical fields of the insular, parietal and temporal regions of primates. J Comp Neurol 168:197-247.

Kosaki H, Hashikawa T, He J, Jones EG (1997) Tonotopic organization of auditory cortical fields delineated by parvalbumin immunoreactivity in macaque monkeys. J Comp Neurol 362:153-170.

LeDoux JE, Farb C, Ruggiero DA (1990) Topographic organization of neurons in the acoustic thalamus that project to the amygdala. J Neurosci 10:1043-1054.

Liu XB, Bolea S, Golshani P, Jones EG (2001) Differentiation of corticothalamic and collateral thalamocortical synapses on mouse reticular nucleus by EPSC amplitude and AMPA receptor subunit composition. Thalamus Related Syst 1:15-29.

Liu X, Warren RA, Jones EG (1995) Synaptic distribution of afferents from reticular nucleus in ventroposterior nucleus of cat thalamus. J Comp Neurol 352:187-202.

McCormick DA, von Krosigk M (1992) Corticothalamic activation modulates thalamic firing through glutamate "metabotropic" receptors. Proc Natl Acad Sci USA 89:2774-2778.

Mitani A, Itoh K, Nomura S, Kudo M, Kaneko T, Mizuno N (1984) Thalamocortical projections to layer I of the primary auditory cortex in the cat: a horseradish peroxidase study. Brain Res 310:347-350.

Mooney DM, Hu B, Senatorov VV (1995) Muscarine induces an anomalous inhibition of synaptic transmission in rat auditory thalamus. J Pharmacol Exp Ther 275:838-844.

Mulle C, Madariaga A, Deschenes M (1986) Morphology and electrophysiological properties of reticularis thalami neurons in cat: in vivo study of a thalamic pacemaker. J Neurosci 6:2134-2145.

Niimi K, Obo K, Kusunose M (1984) Projections of the medial geniculate nucleus to layer 1 of the auditory cortex in the cat traced with horseradish peroxidase. Neurosci Lett 45:223-228.

Ohara PT, Sefton AJ, Lieberman AR (1980) Mode of termination of afferents from the thalamic reticular nucleus in the dorsal lateral geniculate nucleus of the rat. Brain Res 197:503-506.

Palva S, Palva JM, Shtyrov Y, Kujala T, Ilmoniemi RJ, Kaila K, Näätänen R (2002) Distinct gamma-band evoked responses to speech and nonspeech sounds in humans. J Neurosci 22: RC211 (1-5).

Prieto JJ, Peterson BA, Winer JA (1994) Morphology and spatial distribution of GABAergic neurons in cat primary auditory cortex (AI). J Comp Neurol 344:349-382.

Rapisarda C, Bacchelli B (1977) The brain of the guinea pig in stereotaxic coordinates. Arch Sci Biol 61:1-37. 
Rauschecker JP, Tian B, Pons T, Mishikin M (1997) Serial and parallel processing in rhesus monkey auditory cortex. J Comp Neurol 382:89-103.

Redies H, Brandner S, Creutzfeldt OD (1989) Anatomy of the auditory thalamocortical system of the guinea pig. J Comp Neurol 282:489-511.

Ribary U, Ioannides AA, Singh KD, Hasson R, Bolton JPR, Lado F, Mogilner A, Llinás R (1991) Magnetic field tomography of coherent thalamocortical $40-\mathrm{Hz}$ oscillations in humans. Proc Natl Acad Sci USA 88:11037-11041.

Sally SL, Kelly JB (1988) Organization of auditory cortex in the albinos rat: sound frequency. J Neurophysiol 59:1627-1638.

Semple MN, Kitzes LM (1993) Binaural processing of sound pressure level in cat primary auditory cortex: evidence for a representation based on absolute levels rather than interaural level differences. J Neurophysiol 69:449-461.

Shosaku A, Sumitomo I (1983) Auditory neurons in the rat thalamic reticular nucleus. Exp Brain Res 49:432-442.

Shinonaga Y, Tanaka M, Mizuno N (1994) Direct projections from the nonlaminated divisions of the medial geniculate nucleus to the temporal polar cortex and amygdala in the cat. J Comp Neurol 340:405-426.

Sousa-Pinto A (1973) Cortical projections of the medial geniculate body in the cat. Ergeb Anat Entwicklungsgesch 48:1-40.

Steriade M (2001a) Impact of network activities on neuronal properties in corticothalamic systems. J Neurophysiol 86:1-39.

Steriade M (2001b) The GABAergic reticular nucleus: a preferential target of corticothalamic projections. Proc Natl Acad Sci USA 98:3625-3627.

Steriade M, Timofeev I (2003) Neuronal plasticity in thalamocortical network during sleep and waking oscillations. Neuron 37:563-576.

Steriade M, Amzica F, Nunez A (1993a) Cholinergic and noradrenergic modulation of the slow $(\approx 0.3 \mathrm{~Hz})$ oscillation in neocortical cells. J Neurophysiol 70:1384-1400.

Steriade M, Contreras D, Curro Dossi R, Nunez A (1993b) The slow $(<1$ $\mathrm{Hz}$ ) oscillation in reticular thalamic and thalamocortical neurons: Scenario of sleep rhythm generation in interacting thalamic and neocortical networks. J Neurosci 13:3284-3299.

Steriade M, McCormick DA, Sejnowski TJ (1993c) Thalamocortical oscillations in the sleeping and aroused brain. Science 262:679-685.

Steriade M, Nunez A, Amzica F (1993d) A novel slow ( $<1 \mathrm{~Hz}$ ) oscillation of neocortical neurons in vivo: depolarizing and hyperpolarizing components. J Neurosci 13:3252-3265.

Steriade M, Nunez A, Amzica F (1993e) Intracellular analysis of relations between the slow $(<1 \mathrm{~Hz})$ neocortical oscillation and other sleep rhythms of the electroencephalogram. J Neurosci 13:3266-3283.
Steriade M, Timofeev I, Grenier F (2001) Natural waking and sleep states: a view from inside neocortical neurons. J Neurophysiol 85:1969-1985.

Suga N, Horikawa J (1986) Multiple time axes for representation of echo delays in the auditory cortex of the mustached bat. J Neurophysiol 55:776-805.

Terman D, Bose A, Kopell N (1996) Functional reorganization in thalamocortical networks: transition between spindling and delta sleep rhythms. Proc Natl Acad Sci USA 93:15417-15422.

von Krosigk M, Bal T, McCormick DA (1993) Cellular mechanisms of a synchronized oscillation in the thalamus, Science 261:361-364.

Wepsic JG, Sutin J (1964) Posterior thalamic and septal influence upon pallidal and amygdaloid slow wave and unitary activity. Exp Neurol 10:67-80.

Winer JA (1992) The functional architecture of the medial geniculate body and the primary auditory cortex. In: The mammaliam auditory pathway: neuroanatomy (Webster DB, Popper AN, Fay RR, eds), pp 222-409. New York: Springer.

Winer JA, Diamond IT, Raczkowski D (1977) Subdivisions of the auditory cortex of the cat: the retrograde transport of horseradish peroxidase to the medial geniculate and posterior thalamic nuclei. J Comp Neurol 176:387-417

Winer JA, Larue DT (1987) Patterns of reciprocity in auditory thalamocortical and corticothalamic connections: study with horseradish peroxidase and autoradiographic methods in the rat medial geniculate body. J Comp Neurol 257:282-315.

Winer JA, Larue DT (1989) Population of GABAergic neurons and axons in layer I of rat auditory cortex. Neuroscience 33:499-515.

Winer JA, Morest K (1983) The medial division of the medial geniculate body of the cat: Implication for thalamic organization. J Neurosci:2629-2651.

Winer JA, Larue DT, Huang CL (1999) Two systems of giant axon terminals in the cat medial geniculate body: convergence of cortical and GABAergic inputs. J Comp Neurol 413:181-197.

Xiong Y, Yu YQ, Chan YS, He JF (2001) In-vivo intracellular recording on the auditory thalamic neurons. Soc Neurosci Abstr 27:126.

Yan J, Suga N (1996) Corticofugal modulation of time-domain processing of biosonar information in bats. Science 273:1100-1103.

Yen CT, Conley M, Jones EG (1985) The morphology of physiologically identified GABAergic neurons in the somatic sensory part of the thalamic reticular nucleus in the cat. J Neurosci 5:2254-2268.

Zhou X, Jen PHS (2000) Brief and short-term corticofugal modulation of subcortical auditory responses in the big brown bat, eptesicus fuscus. J Neurophysiol 84:3083-3087. 